\section{Shadow of the head of Comet Kohoutek}

HOPKINSON, Elsworth, and James ${ }^{1}$ have reported the detection of a straight dark streak running away from the head of Comet Kohoutek (1973f) for a distance of about $2.5^{\circ}$. They conjectured that the streak could be the shadow of the comet's head as seen in the dust tail of the comet. Their observations were made on January 9, 10,11 , and 12,1974 at about 2200 ur.

We obtained photographic observations of Comet Kohoutek at the Joint Observatory for Cometary Research (JOCR) near Socorro, New Mexico. Wc have observations which overlap in time with those obtained by Hopkinson, Elsworth, and James; a sample taken on January 11, 1974 Ur is shown (Fig. 1). Other JOCR plates have been discussed by Hyder et al. ${ }^{2}$. Our primary plate emulsion was IIa-O, but we also have plates with IIa-E and IIa-F cmulsions. Colour photographs (Ektacolor-L) were also taken.

We feel that the shadow, if real, should be found on our plates, but we have found nothing that we would interpret as the shadow of the head extending $2.5^{\circ}\left(5.4 \times 10^{6} \mathrm{~km}\right)$ in the antisolar direction. At the time of the exposure shown in Fig. 1, the prolonged radius vector was very close to the axis of the plasma tail as projected on the plane of the sky. We have found two features which conceivably could be responsible for the earlier observations ${ }^{1}$. First, a linear dark area was found on a IIa-E plate taken shortly before the IIa-O plate shown in Fig. 1 . This dark streak is in the head, on the side away from the dust tail. Unfortunately, it is neither long enough nor in the correct position to concur with the observations of Hopkinson, Elsworth, and James. In addition, inspection of the Ila-O plate shows clearly that this dark area is just the space between tail rays of the plasma tail. Similar dark streaks pointing toward the nucleus are found on the other side of the head (Fig. 1), but they are not as prominent because the scattered solar radiation from the dust tail tends to fill in the dark spaces. Second, on low resolution photographs the space between the plasma and dust tails could give the appearance of a dark streak.

Whatever the ultimate explanation, we note that our plate scale is $300^{\prime \prime}$ $\mathrm{mm}$ ", and we cstimate that our resolution (which we consider to be caused by plate scale, image quality and cmulsion characteristics) is approximately 10 times greater. The difference in resolution is probably the root of the discrepancy.

Finally, in terms of cometary physics, we believe that observation of the shadow is unlikely for two reasons. First, our colour photographs indicate a low dust content and a relatively weak dust tail as compared with Comet Bennett (1961i). Thus, it is unlikely that observable dust would extend out to $5.4 \times 10^{6} \mathrm{~km} \quad\left(2.5^{\circ}\right)$ except near the axis of the dust tail. Second, the dust tail (presumably composed of micron sized dust) curves away from the antisolar direction. At $5.4 \times 10^{6} \mathrm{~km}$ from the nucleus, the axis of the dust tail lies well away from the antisolar direction.

\section{JOHN C. BRAND'T}

ROBERT G. ROOSEN

Laboratory for Solar Physics and Astrophysics,

NASA-Goddard Space Flight Center, Greenbelt, Maryland 2077 I

${ }^{1}$ Hopkinson, G. R., Elsworth, Y., and James, J. F., Nature, 249, 233 (1974). ${ }^{2}$ Hyder, C. L., Brandt, J. C., and Roosen, R. G., Icarus (in the press).

\section{Platelet monoamine oxidase in schizophrenics}

MURPHy and Wyatt reported reduced monoamine oxidase (MAO) activity in blood platelets from 33 chronic schizophrenic patients ${ }^{1}$ and 13 monozygotic twin pairs ${ }^{2}$ compared with normal controls. They suggested that reduced platelet MAO activity may provide a genetic marker for vulnerability to schizophrenia. We predicted lower MAO activity might sensitise the

Fig. 1 Comet Kohoutek (1973f) on January 11, 1974 ur. This 2 min exposure was taken between $2: 59: 20$ and $3: 01: 20$ UT. The field shown is approximately $3^{\circ} \times 4^{\circ}$.

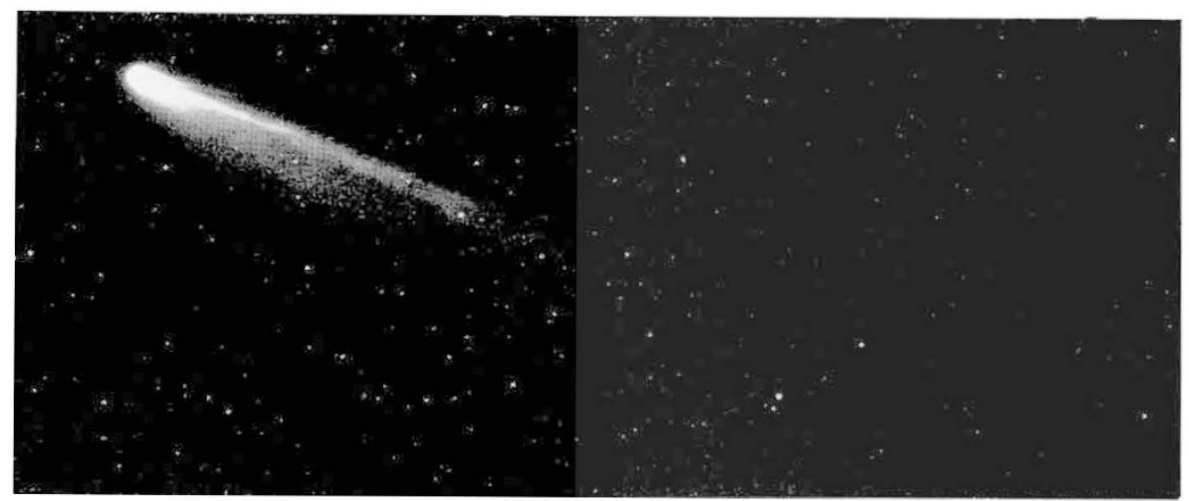

schizophrenic patient to the induction of symptoms by tricyclic antidepressants ${ }^{3}$.

We have completed a study of 24 anergic, depressed schizophrenic outpatients. Patients were free of psychoactive medication for 2 weeks and then blindly treated with either chlorpromazine (Thorazine, SKF) 100-1,200 $\mathrm{mg}$ daily and imipramine (TofranilGeigy) $150 \mathrm{mg}$ daily or thiothixene (Navane-Roerig) 5-60 mg daily and placebo. Platelet MAO activity was measured following the drug-free period and weekly for 4 weeks on medication, using the method of Wyatt and Murphy ${ }^{1,2}$ with ${ }^{14} \mathrm{C}$ tryptamine $\left(1.6 \times 10^{-5} \mathrm{M}\right.$ final concentration) as substrate. Blood samples from eight alcoholic patients and seven staff volunteers served as controls.

We found no differences in platelet MAO activity in the schizophrenic patients compared with alcoholics and volunteers. On the basis of MAO platelet activity (mean \pm standard deviation in nmol product formed per $\mathrm{h}$ per $\mathrm{mg}$ protein) individuals from all three groups could be assigned to either a low $(1.62 \pm 0.34)$ or high $(3.09 \pm 0.53)$ MAO group, with no overlap. Our low MAO group contained 13 schizophrenic patients, four alcoholics and three staff. The high MAO group consisted of 11 patients, four alcoholics and four staff. Discriminant function analysis distinguished high from low MAO level patients on the basis of hyperactivity (Katz adjustment scale factor), sleep disturbance and apathy (Hamilton depression scale factor). No significant differences in primary schizophrenic symptoms existed between patients in the low and high groups at baseline. Preliminary data analysis of 4-week improvement indicates low MAO group patients may do significantly less well on the chlorpromazine-imipramine combination. These findings are under investigation in schizophrenic inpatients.

We have been unable to replicate the reduced blood platelet MAO findings in schizophrenic patients as reported in Nature by Wyatt and Murphy ${ }^{1}$. Individuals have either low or high MAO activity independent of diagnosis. Activity remains stable over at least five week periods. Platelet MAO activity may influence the schizophrenic patient's response to tricyclic medication.

Department of Psychiatry,

EDWARD G. Shaskan

RoBert E. BECKER

University of Connecticut

Health Center,

Farmington, Connecticut 06032

DRS MURPhy aND WyatT REPLYShaskan and Becker's interesting data may be clarified by other recent MAO 